\title{
Performance Comparison of Speaker Identification Using DCT, Walsh, Haar on Full and Row Mean of Spectrogram
}

\author{
Dr. H. B. Kekre \\ Senior Professor, \\ MPSTME, SVKM's \\ NMIMS University \\ Mumbai, 400-056, India.
}

\author{
Dr. T. K. Sarode \\ Assistant Professor, \\ TSEC, Bandra (W), \\ Mumbai, 400-050, India.
}

\author{
Shachi J. Natu \\ Lecturer, TSEC, Bandra \\ (W), Mumbai, 400-050, \\ India.
}

\author{
Prachi J. Natu \\ Assistant Professor, \\ GVAIET, Shelu, Karjat, \\ 410201, India.
}

\begin{abstract}
This paper aims to provide different approaches to text dependent speaker identification using various transformation techniques such as DCT, Walsh and Haar transform along with use of spectrograms. Set of spectrograms obtained from speech samples is used as image database for the study undertaken. This image database is then subjected to various transforms. Using Euclidean distance as measure of similarity, most appropriate speaker match is obtained which is declared to be identified speaker. Each transform is applied to spectrograms in two different ways: on full image and on Row Mean of an image. In both the ways, effect of different number of coefficients of transformed image is observed. Further, comparison of all three transformation techniques on spectrograms in both the ways shows that numbers of mathematical computations required for Walsh transform is much lesser than number of mathematical computations required in case of DCT on spectrograms. Whereas, use of Haar transform on spectrograms drastically reduces the number of mathematical computation with almost equal identification rate. Transformation techniques on Row Mean give better identification rate than transformation technique on full image.
\end{abstract}

Keywords: Speaker identification, Speaker Recognition, Spectrograms, DCT, WALSH, HAAR, Row Mean

\section{INTRODUCTION}

Security has become an extremely important issue due to extensive use of internet technology as well as due to multi-user applications. Identifying users and granting access only to those users who are authorized is a key to provide security. Users can be identified using various approaches and their combinations. As the technology is getting advanced, more sophisticated approaches are being used to satisfy the need of security. Some of the most popular techniques are use of login and password, face recognition, fingerprint recognition; iris recognition etc. Use of login and password is becoming less reliable because of the ease with which attackers can steal the password such as sophisticated electronic eavesdropping techniques [1]. Face recognition, fingerprint recognition and iris recognition also carry their own drawbacks. Users should be willing to undergo the tests and should not get upset by these procedures when these techniques are used to identify them. Speaker identification allows nonintrusive monitoring and also achieves high accuracy rates which conform to most security requirements. Speaker recognition is the process of automatically recognizing who is speaking based on some unique characteristics present in speaker's voice [2]. For this recognition purpose, speaker specific characteristics present in speech signal need to be preserved. Job of Speaker recognition can be classified into two main categories, namely speaker identification and speaker verification. Speaker identification deals with distinguishing a speaker from a group of speakers. In contrast, speaker verification aims to determine if a person is the one who he/she claims to be from a speech sample.

Speaker identification problem can be further classified as text dependent and text independent Speaker Identification based on relevance to speech contents [2]. Text dependent Speaker Identification requires the speaker saying exactly the enrolled or the given password/speech. Text independent Speaker Identification is a process of verifying the identity without constraint on the speech content. Compared to text dependent Speaker Identification, text independent Speaker Identification is more convenient because the user can speak freely to the system. However it requires longer training and testing utterances to achieve good performance.

Speaker Identification task can also be classified into closed set and open set Speaker Identification [3, 4]. In closed set problem, from $\mathrm{N}$ known speakers, the speaker whose reference template has the maximum degree of similarity with the template of input speech sample of unknown speaker is obtained. This unknown speaker is assumed to be one of the given set of speakers. Thus in closed set problem, system makes a forced decision by choosing the best matching speaker from the speaker database. In the open set text dependent speaker identification, matching reference template for an unknown speaker's speech sample may not exist. In this paper, closed set text dependent speaker identification is considered. In the proposed method, speaker identification is carried out with spectrograms and transformation techniques such as DCT, WALSH and HAAR [5, 6, 7, 8]. Thus an attempt is made to formulate a digital signal processing problem into pattern recognition of images.

The rest of the paper is organized as follows: in section 2 we present related work carried out in the field of speaker identification. In section 3 our proposed approach is presented. Section 4 elaborates the experiment conducted. Results are tabulated in section 5. Conclusion has been outlined in section 6 .

\section{RELATED WORK}

Speaker identification problem basically consists of two stages: feature extraction stage and pattern classification stage. In literature there are many approaches available for speaker 
identification process based on various approaches for feature extraction. Feature extraction is the process of extracting subset of features from the entire feature set. The basic idea behind the feature extraction is that the entire feature set is not always necessary for the identification process.

One of the popular approaches for feature extraction is the Mel Frequency Cepstrum Coefficients (MFCC). The MFCC parameter as proposed by Davis and Mermelstein [9] describes the energy distribution of speech signal in a frequency field. Wang Yutai et. al. [10] has proposed a speaker recognition system based on dynamic MFCC parameters. This technique combines the speaker information obtained by MFCC with the pitch to dynamically construct a set of the Mel-filters. These Mel-filters are further used to extract the dynamic MFCC parameters which represent characteristics of speaker's identity.

A histogram based technique was proposed by Sleit, Serhan and Nemir [11] which uses a reduced set of features generated using MFCC method. For these features, histograms are created using predefined interval length. These histograms are generated first for all data in feature set for every speaker. In second approach, histograms are generated for each feature column in feature set of each speaker.

Another widely used method for feature extraction is use of linear Prediction Coefficients (LPC). LPCs capture the information about short time spectral envelope of speech. LPCs represent important speech characteristics such as formant speech frequency and bandwidth [12]. Vector Quantization (VQ) is yet another approach of feature extraction [13, 14, 15, 16, 17]. In Vector Quantization based speaker recognition systems; each speaker is characterized with several prototypes known as code vectors [18]. Speaker recognition based on non-parametric vector quantization was proposed by Pati and Prasanna [19]. Speech is produced due to excitation of vocal tract. In this approach, excitation information can be captured using LP analysis of speech signal and is called as LP residual. This LP residual is further subjected to nonparametric Vector Quantization to generate codebooks of sufficiently large size. Combining nonparametric Vector Quantization on excitation information with vocal tract information obtained by MFCC was also introduced by them.

\section{PROPOSED APPROACH}

In the proposed approach, first the speech samples collected from various speakers have been converted into spectrograms [20]. Spectrograms were created using Short Time Fourier Transfer method as discussed below:

In the approach using STFT, digitally sampled data are divided into chunks of specific size say 128, 256 etc. which usually overlap. Fourier transform is then obtained to calculate the magnitude of the frequency spectrum for each chunk. Each chunk then corresponds to a vertical line in the image, which is a measurement of magnitude versus frequency for a specific moment in time. Thus we converted the speech database into image database. Different transformation techniques such as Discrete Cosine Transform [21, 22, 23], Walsh transform and Haar transform are then applied to these images in two different ways to obtain their feature vectors.

First, every transform is applied on full image [24] and from the feature vectors obtained, different numbers of coefficients were used to identify speaker. Second, transform is applied to Row
Mean of an image to get the feature vector of an image. From this feature vector again identification rate is obtained for various portions selected from the feature vector i.e. for partial feature vector. Out of total database, $80 \%$ of images were used as trainee images and $20 \%$ images were used as test images. Euclidean distance between test image and trainee image is used as a measure of similarity. Euclidean distance between the points $\mathrm{X}\left(\mathrm{X}_{1}, \mathrm{X}_{2}\right.$, etc. $)$ and point $\mathrm{Y}\left(\mathrm{Y}_{1}, \mathrm{Y}_{2}\right.$, etc. $)$ is calculated using the formula shown in equation (1).

$$
\mathrm{D}=\sqrt{\sum_{\mathrm{i}=1}^{\mathrm{n}}\left(X_{i}-Y_{i}\right)^{2}}
$$

Smallest Euclidean distance between test image and trainee image means the most probable match of speaker. Algorithms for transformation technique on full image and transformation techniques on Row Mean of an image are given below.

\subsection{Algorithm for transformation techniques on full image:}

For each trainee image in the database, perform steps 1 to 3.

Step 1:Resize an image to size $256 * 256$.

Step 2:Apply the transformation technique (DCT / WALSH / HAAR) on resized image to obtain its feature vector.

Step 3: Save these feature vectors for further comparison.

Step 4:Read the query image. Repeat step 1 to step 3 for each test image in the database to extract their feature vector.

Step 5:Calculate the Euclidean distance between feature vectors of each test image with each trainee image corresponding to the same sentence.

Step 6: Select the trainee image which has smallest Euclidean distance with the test image and declare the speaker corresponding to this trainee image as the identified speaker.

Step 5 and Step 6 are repeated for selected portion of feature vector obtained by applying Step 2 .

Same steps are repeated except the transformation technique used, to get feature vectors for image database using Walsh and Haar transform.

In the second approach, all three transformation techniques are applied to Row Mean [25] of an image. Row mean is nothing but an average of pixel values of an image along each row. Figure 1 shows how the Row Mean of an image is obtained.

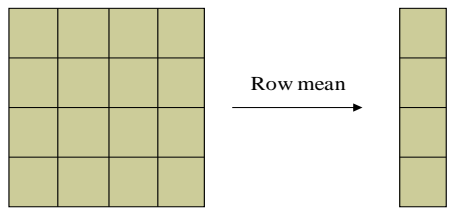

Figure 1: Row Mean of an image

\subsection{Algorithm for transformation technique on row mean of an image}

For each trainee image in the database, perform steps 1 to 3.

Step 1:Resize an image to size $256 * 256$.

Step 2: Calculate Row Mean of an image as shown in Figure 1. 
Step 3:Apply 1-D transformation technique (DCT/WALSH/ HAAR) on Row Mean obtained in Step 2. This gives the feature vector of trainee image.

Step 4: Read query image. Repeat steps 1 to 3 for query image.

Step 5:Apply 1-D transformation technique (DCT / WALSH / HAAR) on Row Mean obtained in Step 2. This gives the feature vector of test image.

Step 6: Calculate the Euclidean distance between feature vectors of test image with each trainee image corresponding to the same sentence.

Step 7: Select the trainee image which has smallest Euclidean distance with the test image and declare the speaker corresponding to this trainee image as the identified speaker.

This Row Mean is obtained for full image. Further, image is divided into four equal and non-overlapping blocks of size $128 * 128$ as shown in Figure 2. Row Mean of each block is calculated and appended to form a feature vector of an image. The most appropriate matching speaker is found out by calculating Euclidean distance between feature vectors of test image and trainee image. The speaker corresponding to the trainee image which has smallest Euclidean distance is declared as the 'Identified speaker'. Similar procedure is repeated by dividing each block obtained using Figure 2 into four equal and nonoverlapping blocks again. This is continued till we get blocks of size $8 * 8$.

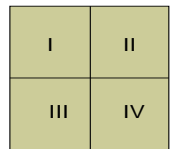

Figure2: Image divided into four equal non-overlapping parts

\section{EXPERIMENTS}

To study the proposed approach we recorded six distinct sentences from 30 speakers: 11 males and 19 females. These sentences are taken from VidTIMIT database [26] and ELSDSR database [27]. For every speaker 10 occurrences of each sentence were recorded. Recording was done at varying times. This forms the closed set for our experiment. From these speech samples spectrograms were created with window size 256 and overlap of 128. Before creation of spectrograms, DC offset present in speech samples was removed so that signals are vertically centered at 0 . After removal of DC offset, speech samples were normalized with respect to amplitude to $-3 \mathrm{~dB}$ and also with respect to time. Spectrograms generated from these speech samples form the image database for our experiment. In all we had 1800 spectrograms in our database.

Eight spectrograms per speaker have been used as trainee images and two spectrograms per speaker as test images. Thus in all we had 1440 spectrograms for training purpose and 360 spectrograms for testing purpose. In the first approach, transformation techniques i.e. DCT, Walsh and Haar were applied on full image to obtain feature vector of image. Later by selecting partial feature vectors, identification rate was obtained. This selection of feature vector is illustrated in following Figure 3 and is based on the number of rows and columns that we selected from the feature vector of an image. For example, we had selected full feature vector (i.e. $256 * 256$ ), then portion of size $192 * 192,128 * 128$, $64 * 64,32 * 32,20 * 20$ and $16 * 16$ was selected from the feature vector. For these different sizes, identification rate was obtained.

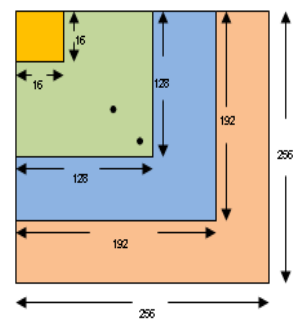

Figure 3: Selection of varying size portion from feature vector

In second approach, Row Mean of these images was calculated and then transformation techniques (DCT, Walsh and Haar) were applied to them to form feature vectors of images. Also images were divided into various sized $(128 * 128,64 * 64,32 * 32, \ldots, 8 * 8)$ equal and non-overlapping blocks. Row Mean of these blocks was calculated to get feature vectors of images. In both approaches, Euclidean distance between feature vectors of test images and trainee images was used as a measure of similarity between images.

Since our work is restricted to text dependent approach, Euclidean distance for a test image of speaker say ' $x$ ' for a particular sentence say ' $\mathrm{s} 1$ ' is obtained by comparing the feature vector of that test image with the feature vectors of all the trainee images corresponding to sentence ' $\mathrm{s} 1$ '. Results are calculated for set of test images corresponding to each sentence.

\section{RESULTS AND COMPLEXITY ANALYSIS \\ 5.1 Results for DCT on spectrograms}

\subsubsection{Results for DCT on full image}

Table 1 show the identification rate for sentences s1 to s6 when different numbers of DCT coefficients are taken to find the matching spectrogram i.e. to identify speaker using DCT on full image. Table 2 shows the overall identification rate considering all sentences, for varying size of portion selected from feature vector. It also shows the number of DCT coefficients used for identifying speaker for corresponding selected portion of feature vector.

Table 1: Identification rate for sentences s1 to $\mathbf{s}$ for varying portion of feature vector when DCT is applied to full image

\begin{tabular}{|c|c|c|c|c|c|c|}
\hline \multirow{2}{*}{$\begin{array}{c}\text { Portion } \\
\text { of } \\
\text { feature } \\
\text { vector } \\
\text { selected }\end{array}$} & S1 & S2 & S3 & S4 & S5 & S6 \\
\hline $256 * 256$ & 63.33 & 66.67 & 75 & 66.67 & 76.67 & 76.67 \\
\hline $192 * 192$ & 73.33 & 70 & 76.67 & 75 & 78.33 & 78.33 \\
\hline $128 * 128$ & 78.33 & 73.33 & 80 & 78.33 & 81.67 & 81.67 \\
\hline $64 * 64$ & 80 & 80 & 78.33 & 86.67 & 83.33 & 88.33 \\
\hline $32 * 32$ & 90 & 86.67 & 86.67 & 86.67 & 86.67 & 90 \\
\hline $20 * 20$ & 86.67 & 86.67 & 86.67 & 88.33 & 90 & 90 \\
\hline $16 * 16$ & 85 & 85 & 86.67 & 86.67 & 91.67 & 90 \\
\hline
\end{tabular}


Table 2: Overall Identification rate for varying number of DCT coefficients when DCT is applied to full image

\begin{tabular}{|c|c|c|}
\hline $\begin{array}{c}\text { Portion of } \\
\text { feature } \\
\text { vector } \\
\text { selected }\end{array}$ & $\begin{array}{c}\text { Number of } \\
\text { DCT } \\
\text { coefficients }\end{array}$ & $\begin{array}{c}\text { Identification } \\
\text { rate (\%) }\end{array}$ \\
\hline $256 * 256$ & 65536 & 70.83 \\
\hline $192 * 192$ & 36864 & 75.27 \\
\hline $128 * 128$ & 16384 & 78.88 \\
\hline $64 * 64$ & 4096 & 82.77 \\
\hline $32 * 32$ & 1024 & 87.77 \\
\hline $20 * 20$ & 400 & $\mathbf{8 8 . 0 5}$ \\
\hline $16 * 16$ & 256 & 87.5 \\
\hline
\end{tabular}

\subsubsection{Results of DCT on row mean of an image}

Table 3 shows sentence wise results obtained when DCT of Row Mean is taken by dividing an image into different number of nonoverlapping blocks. The overall identification rates when DCT of Row Mean is taken by dividing an image into different number of non-overlapping blocks in Table 4 .

Table 3: Identification rate for sentences s1 to s6 for DCT on Row mean of an image when image is divided into different number of non-overlapping and equal sized blocks

\begin{tabular}{|c|c|c|c|c|c|c|}
\hline $\begin{array}{c}\text { No. of blocks } \\
\text { for } \\
\text { image split }\end{array}$ & $\mathbf{S 1}$ & $\mathbf{S 2}$ & $\mathbf{S 3}$ & $\mathbf{S 4}$ & $\mathbf{S 5}$ & $\mathbf{S 6}$ \\
\cline { 2 - 7 } & \multicolumn{6}{|c|}{ Sentence } \\
\hline $\begin{array}{c}\text { Full image } \\
(256 * 256)\end{array}$ & 73.33 & 76.67 & 78.33 & 76.67 & 75 & 80 \\
\hline $\begin{array}{c}4 \text { Blocks } \\
(128 * 128)\end{array}$ & 80 & 80 & 78.33 & 81.67 & 81.67 & 80 \\
\hline $\begin{array}{c}16 \text { Blocks } \\
(64 * 64)\end{array}$ & 91.67 & 81.67 & 83.33 & 83.33 & 81.67 & 83.33 \\
\hline $\begin{array}{c}64 \text { Blocks } \\
(32 * 32)\end{array}$ & 91.67 & 85 & 86.67 & 86.67 & 86.67 & 88.33 \\
\hline $\begin{array}{c}256 \text { Blocks } \\
(16 * 16)\end{array}$ & 91.67 & 88.33 & 88.33 & 85 & 91.67 & 90 \\
\hline $\begin{array}{c}1024 \text { Blocks } \\
(8 * 8)\end{array}$ & 88.33 & 83.33 & 85 & 86.67 & 85 & 88.33 \\
\hline
\end{tabular}

Table 4: Overall Identification rate for DCT on Row mean of an image when image is divided into different number of nonoverlapping blocks of equal size

\begin{tabular}{|c|c|c|}
\hline $\begin{array}{c}\text { No. of blocks for } \\
\text { Image split }\end{array}$ & $\begin{array}{c}\text { Number of } \\
\text { DCT } \\
\text { Coefficients }\end{array}$ & $\begin{array}{c}\text { Identification } \\
\text { Rate (\%) }\end{array}$ \\
\hline Full image $(256 * 256)$ & 256 & 76.67 \\
\hline 4 Blocks $(128 * 128)$ & 512 & 80.27 \\
\hline 16 Blocks $(64 * 64)$ & 1024 & 84.17 \\
\hline 64 Blocks $(32 * 32)$ & 2048 & 87.5 \\
\hline 256 Blocks $(16 * 16)$ & 4096 & $\mathbf{8 9 . 1 7}$ \\
\hline 1024 Blocks $(8 * 8)$ & 8192 & 86.11 \\
\hline
\end{tabular}

\subsection{Results Of Walsh Transform On Spectrograms}

\subsubsection{Results for Walsh on full image}

Results of Walsh transform on Spectrograms are tabulated below. Table 5 shows the identification rate for sentences s1 to s6 when different numbers of Walsh transform's coefficients are taken to find the matching spectrogram i.e. to identify speaker using first approach. Table 6 shows the overall identification rate considering all sentences, for various percentages of Walsh coefficients i.e. for partial feature vectors.

Table 5: Identification rate for sentences $s 1$ to $\mathbf{s} 6$ for varying portion of feature vector when Walsh transform is applied to full image

\begin{tabular}{|c|c|c|c|c|c|c|}
\hline \multirow{2}{*}{$\begin{array}{c}\text { Portion } \\
\text { of } \\
\text { feature } \\
\text { vector } \\
\text { selected }\end{array}$} & \multicolumn{6}{|c|}{ Sentence } \\
\hline & S1 & S2 & S3 & S4 & S5 & S6 \\
\hline $256 * 256$ & 63.33 & 66.67 & 75 & 66.67 & 76.67 & 76.67 \\
\hline $192 * 192$ & 75 & 71.67 & 76.67 & 73.33 & 78.33 & 81.67 \\
\hline $128 * 128$ & 80 & 75 & 78.33 & 83.33 & 81.67 & 81.67 \\
\hline $64 * 64$ & 86.67 & 83.33 & 81.67 & 85 & 83.33 & 85 \\
\hline $32 * 32$ & 86.67 & 81.67 & 81.67 & 88.33 & 83.33 & 91.67 \\
\hline $20 * 20$ & 91.67 & 78.33 & 83.33 & 85 & 86.67 & 83.33 \\
\hline $16 * 16$ & 86.67 & 85 & 83.33 & 85 & 83.33 & 86.67 \\
\hline
\end{tabular}

Table 6: Overall Identification rate for varying number of coefficients when Walsh is applied to full image

\begin{tabular}{|c|c|c|}
\hline $\begin{array}{c}\text { Portion of feature } \\
\text { vector selected }\end{array}$ & $\begin{array}{c}\text { Number of } \\
\text { Walsh } \\
\text { Coefficients }\end{array}$ & $\begin{array}{c}\text { Identification } \\
\text { rate (\%) }\end{array}$ \\
\hline $256 * 256$ & 65536 & 70.83 \\
\hline $192 * 192$ & 36864 & 76.11 \\
\hline $128 * 128$ & 16384 & 80 \\
\hline $64 * 64$ & 4096 & 84.16 \\
\hline $32 * 32$ & 1024 & $\mathbf{8 5 . 5 5}$ \\
\hline $20 * 20$ & 400 & 84.72 \\
\hline $16 * 16$ & 256 & 85 \\
\hline
\end{tabular}

\subsubsection{Results of Walsh on row mean of an image}

Table 7 shows the sentencewise identification rate identification rate when Walsh transform is applied to Row Mean of an image when it is divided into different number of non-overlapping blocks.

Whereas Table 8 shows the overall identification rate by considering the identification rate of all the sentences, when Walsh transform is applied to Row Mean of an image and to the Row Mean of image blocks. These image blocks are obtained by dividing an image into different number of non-overlapping blocks. 
Table 7: Identification rate for sentences s1 to s6 for Walsh transform on Row mean of an image when image is divided into different number of non-overlapping blocks

\begin{tabular}{|c|c|c|c|c|c|c|}
\hline \multirow{2}{*}{$\begin{array}{l}\text { No. of blocks } \\
\text { for } \\
\text { image split }\end{array}$} & \multicolumn{6}{|c|}{ Sentence } \\
\hline & S1 & $\mathbf{S 2}$ & S3 & S4 & S5 & S6 \\
\hline $\begin{array}{l}\text { Full image } \\
(256 * 256)\end{array}$ & 73.33 & 76.66 & 78.33 & 76.66 & 75 & 80 \\
\hline $\begin{array}{l}\text { 4 Blocks } \\
(128 * 128)\end{array}$ & 80 & 80 & 78.33 & 81.67 & 81.67 & 80 \\
\hline $\begin{array}{l}16 \text { Blocks } \\
(64 * 64)\end{array}$ & 91.67 & 81.67 & 83.33 & 83.33 & 81.67 & 83.33 \\
\hline $\begin{array}{c}64 \text { Blocks } \\
(32 * 32)\end{array}$ & 91.67 & 85 & 86.66 & 86.66 & 86.66 & 88.33 \\
\hline $\begin{array}{c}256 \text { Blocks } \\
(16 * 16)\end{array}$ & 91.67 & 88.33 & 88.33 & 85 & 91.67 & 90 \\
\hline $\begin{array}{c}1024 \text { Blocks } \\
(8 * 8)\end{array}$ & 88.33 & 83.33 & 85 & 86.66 & 85 & 88.33 \\
\hline
\end{tabular}

Table 8: Overall Identification rate for Walsh transform on Row mean of an image when image is divided into different number of non-overlapping blocks of equal size

\begin{tabular}{|c|c|c|}
\hline $\begin{array}{c}\text { No. of blocks for } \\
\text { image split }\end{array}$ & $\begin{array}{c}\text { Number of } \\
\text { Walsh } \\
\text { coefficients }\end{array}$ & $\begin{array}{c}\text { Identification } \\
\text { Rate (\%) }\end{array}$ \\
\hline Full image $(256 * 256)$ & 256 & 76.67 \\
\hline 4 Blocks $(128 * 128)$ & 512 & 80.27 \\
\hline 16 Blocks $(64 * 64)$ & 1024 & 84.17 \\
\hline 64 Blocks $(32 * 32)$ & 2048 & 87.5 \\
\hline 256 Blocks $(16 * 16)$ & 4096 & $\mathbf{8 9 . 1 7}$ \\
\hline 1024 Blocks $(8 * 8)$ & 8192 & 86.11 \\
\hline
\end{tabular}

5.3 Results Of Haar Transform On Spectro-grams

\subsubsection{Results for Haar on full image}

Table 9 shows sentencewise identification rate when 2-D Haar transform is applied to full image and different numbers of coefficients are taken. Overall identification rate for Haar transform on full image and for its partial coefficients is shown in Table 10.

Table 9: Identification rate for sentences s1 to s6 for varying portion of feature vector when Haar transform is applied to full image

\begin{tabular}{|c|c|c|c|c|c|c|}
\hline \multirow{2}{*}{$\begin{array}{c}\text { Portion } \\
\text { of } \\
\text { feature } \\
\text { vector } \\
\text { selected }\end{array}$} & \multicolumn{7}{|c|}{ Sentence } \\
\cline { 2 - 8 } & S1 & S2 & S3 & S4 & S5 & S6 \\
\hline $256 * 256$ & 63.33 & 66.67 & 75 & 66.67 & 76.67 & 76.67 \\
\hline $192 * 192$ & 80 & 73.33 & 78.33 & 76.67 & 78.33 & 78.33 \\
\hline $128 * 128$ & 80 & 75 & 78.33 & 83.33 & 81.67 & 81.67 \\
\hline $64 * 64$ & 86.67 & 83.33 & 81.67 & 85 & 83.33 & 85 \\
\hline $32 * 32$ & 86.67 & 81.67 & 81.67 & 88.33 & 83.33 & 91.67 \\
\hline $20 * 20$ & 86.67 & 88.33 & 86.67 & 85 & 85 & 86.67 \\
\hline $16 * 16$ & 86.67 & 85 & 83.33 & 85 & 83.33 & 86.67 \\
\hline
\end{tabular}

Table 10: Overall Identification rate for Haar transform on full image

\begin{tabular}{|c|c|c|}
\hline $\begin{array}{c}\text { Portion of } \\
\text { feature } \\
\text { vector selected }\end{array}$ & $\begin{array}{c}\text { Number of HAAR } \\
\text { Coefficients }\end{array}$ & $\begin{array}{c}\text { Identification } \\
\text { rate }(\boldsymbol{\%})\end{array}$ \\
\hline $256 * 256$ & 65536 & 70.83 \\
\hline $192 * 192$ & 36864 & 77.5 \\
\hline $128 * 128$ & 16384 & 80 \\
\hline $64 * 64$ & 4096 & 84.16 \\
\hline $32 * 32$ & 1024 & 85.55 \\
\hline $20 * 20$ & 400 & $\mathbf{8 6 . 3 9}$ \\
\hline $16 * 16$ & 256 & 85 \\
\hline
\end{tabular}

\subsubsection{Results for Haar on row mean of an image}

Table 11 shows identification rate for each sentence when 1-D Haar transform was applied on Row Mean of images divided into different number of non-overlapping and equal sized blocks

Table 11: Identification rate for sentences s1 to s6 for Haar transform on Row mean of an image when image is divided into different number of non-overlapping blocks

\begin{tabular}{|c|c|c|c|c|c|c|}
\hline \multirow{2}{*}{$\begin{array}{l}\text { No. of blocks } \\
\text { for } \\
\text { image split }\end{array}$} & \multicolumn{6}{|c|}{ Sentence } \\
\hline & S1 & $\mathbf{S 2}$ & S3 & S4 & S5 & S6 \\
\hline $\begin{array}{l}\text { Full image } \\
(256 * 256)\end{array}$ & 73.33 & 76.67 & 78.33 & 76.67 & 75 & 80 \\
\hline $\begin{array}{c}\text { 4 Blocks } \\
(128 * 128)\end{array}$ & 80 & 80 & 78.33 & 81.67 & 81.67 & 80 \\
\hline $\begin{array}{l}16 \text { Blocks } \\
(64 * 64)\end{array}$ & 91.67 & 81.67 & 83.33 & 83.33 & 81.67 & 83.33 \\
\hline $\begin{array}{l}64 \text { Blocks } \\
(32 * 32)\end{array}$ & 91.67 & 85 & 86.67 & 86.67 & 86.67 & 88.33 \\
\hline $\begin{array}{c}256 \text { Blocks } \\
(16 * 16)\end{array}$ & 91.67 & 88.33 & 88.33 & 85 & 91.67 & 90 \\
\hline $\begin{array}{c}1024 \text { Blocks } \\
(8 * 8)\end{array}$ & 88.33 & 83.33 & 85 & 86.67 & 85 & 88.33 \\
\hline
\end{tabular}

Overall identification rate for Haar transform on Row Mean of images divided into different number of equal and nonoverlapping blocks for two training sets is shown in Table 12.

Table 12: Overall Identification rate for Haar transform on Row mean of an image when image is divided into different number of non-overlapping and equal sized blocks

\begin{tabular}{|c|c|c|}
\hline $\begin{array}{c}\text { No. of blocks for } \\
\text { image split }\end{array}$ & $\begin{array}{c}\text { Number of Haar } \\
\text { coefficients }\end{array}$ & $\begin{array}{c}\text { Identification } \\
\text { Rate (\%) }\end{array}$ \\
\hline Full image $(256 * 256)$ & 256 & 76.67 \\
\hline 4 Blocks $(128 * 128)$ & 512 & 80.27 \\
\hline 16 Blocks $(64 * 64)$ & 1024 & 84.17 \\
\hline 64 Blocks $(32 * 32)$ & 2048 & 87.5 \\
\hline 256 Blocks $(16 * 16)$ & 4096 & $\mathbf{8 9 . 1 7}$ \\
\hline 1024 Blocks $(8 * 8)$ & 8192 & 86.11 \\
\hline
\end{tabular}

\subsection{Complexity Analysis}

For 2-D DCT on $\mathrm{N}^{*} \mathrm{~N}$ image, $2 \mathrm{~N}^{3}$ multiplications are required and $2 \mathrm{~N}^{2}(\mathrm{~N}-1)$ additions are required. For $2-\mathrm{D}$ Walsh on $\mathrm{N}^{*} \mathrm{~N}$ image, $2 \mathrm{~N}^{2}(\mathrm{~N}-1)$ additions are required. For 2-D Haar transform on $\mathrm{N}^{*} \mathrm{~N}$ image where $\mathrm{N}=2^{\mathrm{m}}$, number of multiplications required are $(\mathrm{m}+1) \mathrm{N}$ and number of additions required are $2 \mathrm{mN}^{2}$. For $1-\mathrm{D}$ 
DCT on $1 * \mathrm{~N}$ image, $\mathrm{N}^{2}$ multiplications are needed and $\mathrm{N}(\mathrm{N}-1)$ additions are needed. For 1-D WALSH on $1 * \mathrm{~N}$ image, $\mathrm{N}(\mathrm{N}-1)$ additions are needed. For 1-D Haar transform on $\mathrm{N}^{*} 1$ image, number of multiplications required are $(\mathrm{m}+1) \mathrm{N}$ and number of additions are $\mathrm{mN}$. These details are summarized in table 13 .

Table 13: Computational details for 2-D DCT on full $\mathbf{N}^{*} \mathbf{N}$ image, 2-D Walsh on full $N * N$ image, 2-D Haar on full $N^{*} N$ image, 1-D DCT on $N^{*} 1$ Row Mean of an image, 1-D Walsh on $N^{* 1} 1$ Row Mean of an image and 1-D Haar on $N^{*} 1$ Row Mean of an image respectively.

\begin{tabular}{|c|c|c|c|c|c|c|}
\hline \multirow[b]{2}{*}{ 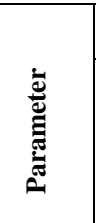 } & \multicolumn{6}{|c|}{ Algorithm } \\
\hline & $\begin{array}{l}\text { DCT } \\
\text { on full } \\
\text { image }\end{array}$ & $\begin{array}{l}\text { Walsh } \\
\text { on full } \\
\text { image }\end{array}$ & $\begin{array}{c}\text { Haar on } \\
\text { full } \\
\text { image }\end{array}$ & $\begin{array}{l}\text { DCT } \\
\text { on } \\
\text { Row } \\
\text { Mean }\end{array}$ & $\begin{array}{l}\text { Wals } \\
\text { h on } \\
\text { Row } \\
\text { Mean }\end{array}$ & $\begin{array}{c}\text { Haar } \\
\text { on } \\
\text { Row } \\
\text { Mean }\end{array}$ \\
\hline 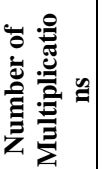 & $2 \mathrm{~N}^{3}$ & 0 & $2(m+1) * N^{2}$ & $\mathrm{~N}^{2}$ & 0 & $\begin{array}{c}(\mathrm{m}+1) \\
{ }^{*} \mathrm{~N}\end{array}$ \\
\hline 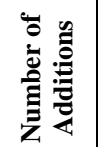 & $\begin{array}{l}2 \mathrm{~N}^{2} * \\
(\mathrm{~N}-1)\end{array}$ & $\begin{array}{l}2 \mathrm{~N}^{2} * \\
(\mathrm{~N}-1)\end{array}$ & $2 \mathrm{mN}^{2}$ & $\begin{array}{c}\mathrm{N}^{*} \\
(\mathrm{~N}-1)\end{array}$ & $\begin{array}{c}\mathrm{N}^{*} \\
(\mathrm{~N}-1)\end{array}$ & $\mathrm{mN}$ \\
\hline
\end{tabular}

Table 14 shows comparison of number of multiplications required, number of additions required and identification rate when DCT, Walsh and Haar are applied on full image of size $256 * 256$ and on Row Mean of an image.

Table 14: Number of multiplications and number of additions for DCT on full image, 2-D Walsh on full image, 2-D Haar on full image, 1-D DCT on Row Mean of an image, 1-D Walsh on Row Mean of an image and 1-D Haar on Row Mean of an image of size $256 * 256$.

\begin{tabular}{|c|c|c|c|c|c|c|}
\hline \multirow[b]{2}{*}{ Parameter } & \multicolumn{6}{|c|}{ Algorithm } \\
\hline & $\begin{array}{l}\text { DCT } \\
\text { on full } \\
\text { image }\end{array}$ & $\begin{array}{l}\text { Walsh } \\
\text { on full } \\
\text { image }\end{array}$ & $\begin{array}{l}\text { Haar } \\
\text { on full } \\
\text { image }\end{array}$ & $\begin{array}{c}\text { DCT on } \\
\text { Row } \\
\text { Mean }\end{array}$ & $\begin{array}{l}\text { Walsh } \\
\text { on } \\
\text { Row } \\
\text { Mean }\end{array}$ & $\begin{array}{l}\text { Haar } \\
\text { on } \\
\text { Row } \\
\text { Mean }\end{array}$ \\
\hline $\begin{array}{l}\text { Number of } \\
\text { multiplicatio } \\
\text { ns }\end{array}$ & $3.3 * 10^{7}$ & 0 & $1.1 * 10^{6}$ & $6.5^{*} 10^{4}$ & 0 & $\begin{array}{c}2.3^{*} \\
10^{3}\end{array}$ \\
\hline $\begin{array}{l}\text { Number of } \\
\text { additions }\end{array}$ & $3.3 * 10^{7}$ & $3.3 * 10^{7}$ & $10^{6}$ & $6.5 * 10^{4}$ & $\begin{array}{c}6.5^{*} \\
10^{4}\end{array}$ & $2 * 10^{3}$ \\
\hline $\begin{array}{l}\text { Identification } \\
\text { rate }(\%)\end{array}$ & 88.05 & 85.55 & 86.39 & 89.17 & 89.17 & 89.17 \\
\hline
\end{tabular}

\section{CONCLUSION}

In this paper we considered closed set text dependent speaker identification using three different transformation techniques: DCT, Walsh and Haar. For each transformation technique identification rate was obtained using two approaches. First, by applying transformation technique on full image and second, by applying transformation technique on Row Mean of an image by dividing image into different number of equal sized and nonoverlapping blocks. It has been observed that in the first approach, as the number of coefficients chosen is smaller up to a certain limit; better identification rate is achieved in all three transformation techniques. DCT on full image gives its best identification rate of $88.05 \%$ when $20 * 20$ portion of feature vector is selected i.e. for 400 DCT coefficients. Walsh transform gives its maximum identification rate $85.55 \%$ for $32 * 32$ portion of feature vector whereas, Haar transform gives maximum identification rate for feature vector portion of size $20 * 20$.

When transformation techniques on full image are compared as shown in table 14, it can be observed that for Walsh transform on full image, numbers of mathematical computations required are greatly reduced as compared to DCT since no multiplications are required in Walsh. These computations are further reduced by use of Haar transform. DCT on full image requires 28 times more multiplications and 32 times more additions as compared to Haar transform on full image. Walsh transform on full image 32 times more additions as compared to Haar transform on full image. Though the number of multiplications required in Walsh transform on full image is zero, total CPU time required by Haar transform is less than that of Walsh transform. Better identification rate is achieved by DCT as compared to Walsh and Haar transform but at the expense of higher number of mathematical computations.

Similarly, when transformation techniques on Row Mean of an image are compared, it can be clearly seen from table 14 that, Haar transform on Row Mean of an image gives same identification rate with less number of computations as compared to DCT on Row Mean of an image and Walsh on Row Mean of an image. DCT on Row Mean of an image requires 28 times of multiplications and 32 times more additions as compared to Haar transform on full image. Again here though the number of multiplications required in Walsh transform on full image is zero, because of large number of additions, it requires more CPU time.

From overall comparison shown in table 14, we can conclude that Row Mean technique requires less number of mathematical computations and hence less CPU time for all three transformation techniques as compared to transformation techniques on full image. Haar transform on Row Mean of an image gives the best result with respect to identification rate as well as number of mathematical computations required.

\section{REFERENCES}

[1] Evgeniy Gabrilovich, Alberto D. Berstin: "Speaker recognition: using a vector quantization approach for robust text-independent speaker identification", Technical report DSPG-95-9-001', September 1995.

[2] Tridibesh Dutta, "Text dependent speaker identification based on spectrograms", Proceedings of Image and vision computing, pp. 238-243, New Zealand 2007.

[3] J.P.Campbell, "Speaker recognition: a tutorial", Proc. IEEE, vol. 85, no. 9, pp. 1437-1462, 1997.

[4] D. O'Shaughnessy, "Speech communications- Man and Machine”, New York, IEEE Press, 2nd Ed., pp. 199, pp. 437458,2000 . 
[5] H.B.Kekre, Sudeep D. Thepade, "Improving the Performance of Image Retrieval using Partial Coefficients of Transformed Image", International Journal of Information Retrieval (IJIR), Serials Publications, Volume 2, Issue 1, pp. $72-79$ (ISSN: 0974-6285), 2009.

[6] H.B.Kekre, Tanuja Sarode, Sudeep D. Thepade, "DCT Applied to Row Mean and Column Vectors in Fingerprint Identification", In Proceedings of International Conference on Computer Networks and Security (ICCNS), 27-28 Sept. 2008, VIT, Pune.

[7] H.B.Kekre, Sudeep D. Thepade, Archana Athawale, Anant Shah, Prathmesh Verlekar, Suraj Shirke,"Energy Compaction and Image Splitting for Image Retrieval using Kekre's Transform over Row and Column Feature Vectors", International Journal of Computer Science and Network Security (IJCSNS),Volume:10, Number 1, January 2010, (ISSN: 1738-7906) Available at www.IJCSNS.org.

[8] H.B.Kekre, Sudeep D. Thepade, Archana Athawale, Anant Shah, Prathmesh Verlekar, Suraj Shirke, "Performance Evaluation of Image Retrieval using Energy Compaction and Image Tiling over DCT Row Mean and DCT Column Mean", Springer-International Conference on Contours of Computing Technology (Thinkquest-2010), Babasaheb Gawde Institute of Technology, Mumbai, 13-14 March 2010, The paper will be uploaded on online Springerlink.

[9] S. Davis and P. Mermelstein, "Comparison of parametric representations for monosyllabic word recognition in continuously spoken sentences," IEEE Transaction Acoustics Speech and Signal Processing, vol. 4, pp. 375-366, 1980.

[10] Wang Yutai, Li Bo, Jiang Xiaoqing, Liu Feng, Wang Lihao, "Speaker Recognition Based on Dynamic MFCC Parameters", International Conference on Image Analysis and Signal Processing, pp. 406-409, 2009

[11] Azzam Sleit, Sami Serhan, and Loai Nemir, "A histogram based speaker identification technique", International Conference on ICADIWT, pp. 384-388, May 2008.

[12] B. S. Atal, "Automatic Recognition of speakers from their voices”, Proc. IEEE, vol. 64, pp. 460-475, 1976.

[13] H. B. Kekre, Tanuja K. Sarode, Sudeep D. Thepade, "Image Retrieval by Kekre's Transform Applied on Each Row of Walsh Transformed VQ Codebook", (Invited), ACMInternational Conference and Workshop on Emerging Trends in Technology (ICWET 2010), Thakur College of Engg. And Tech., Mumbai, 26-27 Feb 2010, the paper is invited at ICWET 2010. Also will be uploaded on online ACM Portal.

[14] H. B. Kekre, Tanuja Sarode, Sudeep D. Thepade, "ColorTexture Feature based Image Retrieval using DCT applied on Kekre's Median Codebook", International Journal on Imaging (IJI), Volume 2, Number A09, Autumn 2009,pp. 5565. Available online at www.ceser.res.in/iji.html (ISSN: 0974-0627).

[15] H. B. Kekre, Ms. Tanuja K. Sarode, Sudeep Thepade, "Image Retrieval using Color-Texture Features from DCT on VQ Codevectors obtained by Kekre's Fast Codebook Generation", ICGST-International Journal on Graphics, Vision and Image Processing (GVIP), Volume 9, Issue 5, pp.: 1-8, September 2009. Available online at http: //www.icgst.com/gvip/Volume9/Issue5/P1150921752.html.
[16] H. B. Kekre, Tanuja Sarode "Two Level Vector Quantization Method for Codebook Generation using Kekre's Proportionate Error Algorithm" , CSC-International Journal of Image Processing, Vol.4, Issue 1, pp.1-10, JanuaryFebruary 2010

[17] H.B.Kekre, Tanuja K. Sarode, Sudeep D. Thepade, Vaishali Suryavanshi, "Improved Texture Feature Based Image Retrieval using Kekre's Fast Codebook Generation Algorithm", Springer-International Conference on Contours of Computing Technology (Thinkquest-2010), Babasaheb Gawde Institute of Technology, Mumbai, 13-14 March 2010, The paper will be uploaded on online Springerlink.

[18] Jialong He, Li Liu, and G"unther Palm, "A discriminative training algorithm for VQ-based speaker Identification", IEEE Transactions on speech and audio processing, vol. 7, No. 3, pp. 353-356, May 1999.

[19] Debadatta Pati, S. R. Mahadeva Prasanna, "Non-Parametric Vector Quantization of Excitation Source Information for Speaker Recognition", IEEE Region 10 Conference, pp. 1-4, Nov. 2008.

[20] Tridibesh Dutta and Gopal K. Basak, "Text dependent speaker identification using similar patterns in spectrograms", PRIP'2007 Proceedings, Volume 1, pp. 8792, Minsk, 2007.

[21] Andrew B. Watson, "Image compression using the Discrete Cosine Transform", Mathematica journal, 4(1), pp. 81-88, 1994.

[22] H. B. Kekre, Sudeep Thepade, Akshay Maloo, "Image Retrieval using Fractional Coefficients of Transformed Image using DCT and Walsh Transform", International Journal of Engineering Science and Technology, Vol.. 2, No. 4, 2010, 362-371

[23] H. B. Kekre, Sudeep Thepade and Akshay Maloo, "Performance Comparison of Image Retrieval Using Fractional Coefficients of Transformed Image Using DCT, Walsh, Haar and Kekre's Transform", CSC-International Journal of Image processing (IJIP), Vol.. 4, No.2, pp.:142155, May 2010.

[24] H. B. Kekre, Tanuja Sarode, Shachi Natu, Prachi Natu, "Performance Comparison Of 2-D DCT On Full/Block Spectrogram And 1-D DCT On Row Mean Of Spectrogram For Speaker Identification", (Selected) CSC-International Journal of Biometrics and Bioinformatics (IJBB), Volume (4): Issue (3).

[25] H. B. Kekre, Sudeep Thepade, Akshay Maloo, "Eigenvectors of Covariance Matrix using Row Mean and Column Mean Sequences for Face Recognition", CSC-International Journal of Biometrics and Bioinformatics (IJBB), Volume (4): Issue (2), pp. 42-50, May 2010.

[26] http://www.itee.uq.edu.au/ conrad/vidtimit/

[27] http://www2.imm.dtu.dk/ lf/elsdsr/

\section{AUTHOR BIOGRAPHIES}

Dr. H. B. Kekre has received B.E. (Hons.) in Telecomm. Engg. from Jabalpur University in 1958, M.Tech (Industrial Electronics) from IIT Bombay in 1960, M.S.Engg. (Electrical Engg.) from University of Ottawa in 1965 and Ph.D. (System Identification) 
from IIT Bombay in 1970. He has worked Over 35 years as Faculty of Electrical Engineering and then HOD Computer Science and Engg. at IIT Bombay. For last 13 years worked as a Professor in Department of Computer Engg. at Thadomal Shahani Engineering College, Mumbai. He is currently Senior Professor working with Mukesh Patel School of Technology Management and Engineering, SVKM's NMIMS University, Vile Parle(w), Mumbai, INDIA. He ha guided 17 Ph.D.s, 150 M.E./M.Tech Projects and several B.E./B.Tech Projects. His areas of interest are Digital Signal processing, Image Processing and Computer Networks. He has more than 250 papers in National / International Conferences / Journals to his credit. Recently six students working under his guidance have received best paper awards. Currently he is guiding ten $\mathrm{Ph}$.D. students.

Dr. Tanuja K. Sarode has received M.E.(Computer Engineering) degree from Mumbai University in 2004 and Ph.D. from Mukesh Patel School of Technology, Management and Engg., SVKM's NMIMS University, Vile-Parle (W), Mumbai, INDIA in 2010. She has more than 10 years of experience in teaching. Currently working as Assistant Professor in Dept. of Computer Engineering at Thadomal Shahani Engineering College, Mumbai. She is member of International Association of Engineers (IAENG) and
International Association of Computer Science and Information Technology (IACSIT). Her areas of interest are Image Processing, Signal Processing and Computer Graphics. She has 70 papers in National /International Conferences/journal to her credit.

Shachi Natu has received B.E.(Computer) degree from Mumbai University with first class in 2004. Currently pursuing M.E. in Computer Engineering from University of Mumbai. She has 05 years of experience in teaching. Currently working as Lecturer in department of Information Technology at Thadomal Shahani Engineering College, Bandra (w), Mumbai. Her areas of interest are Image Processing, Data Structure, Database Management Systems and operating systems.

Prachi Natu has received B.E.(Electronics and Telecommunication) degree from Mumbai University with first class in 2004. Currently pursuing M.E. in Computer Engineering from University of Mumbai. She has 04 years of experience in teaching. Currently working as Assistant Professor in Department of Computer Engineering at G. V. Acharya Institute of Engineering and Technology, Shelu, Karjat. Her areas of interest are Image Processing, Database Management Systems and operating systems. 